\title{
Paediatric musculoskeletal US beyond the hip joint
}

\author{
Carlo Martinoli • Maura Valle • Clara Malattia • \\ Maria Beatrice Damasio • Alberto Tagliafico
}

Received: 5 January 2011 / Accepted: 14 January 2011

(C) Springer-Verlag 2011

\begin{abstract}
US is a technique particularly suited to the investigation of musculoskeletal disorders in children and adolescents. This review paper describes a range of clinical settings beyond the hip joint where US has a significant role to play, including sports injuries, infectious diseases, inflammatory and degenerative conditions, congenital and developmental disorders, acute trauma of bone and joints, and peripheral nerve injuries. In some circumstances, US can be regarded as the most effective means of diagnostic imaging, whereas in other instances, it is an alternative or supplement to other more comprehensive imaging modalities, like MRI and CT. Although MRI offers superior soft-tissue contrast resolution, US is low-cost, non-invasive and has higher spatial resolution and real-time capability for the assessment of musculoskeletal structures during joint movement and stress manoeuvres.
\end{abstract}

Disclaimer The supplement this article is part of is not sponsored by the industry. Dr. Carlo Martinoli, Maura Valle, Clara Malattia, Maria Beatrice Damasio and Alberto Tagliafico has no financial interests, investigational or off-label uses to disclose.

C. Martinoli $(\bowtie)$

Radiologia - DISC, Università di Genova,

Largo Rosanna Benzi 8,

16132 Genoa, Italy

e-mail: carlo.martinoli@libero.it

M. Valle $\cdot$ M. Beatrice Damasio

Radiologia, IRCCS Gaslini Children Hospital,

Genoa, Italy

\section{Malattia}

Pediatria II, Reumatologia, IRCCS Gaslini Children Hospital, Genoa, Italy

\section{A. Tagliafico}

Radiologia, National Institute for Cancer Research,

Genoa, Italy
Keywords Ultrasound - Musculoskeletal system · Trauma $\cdot$ Child

High-resolution US is particularly well suited to examine the paediatric musculoskeletal system. An exquisite depiction of the immature skeleton and soft tissues can be obtained in a well-tolerated and non-invasive way without using ionising radiation or sedation. Although US has been widely used in the diagnosis of hip disorders in children, many other applications for US of the paediatric musculoskeletal system have been developed $[1,2]$. Over other imaging techniques, US is ideally suited for the evaluation of tendons, muscles, joints, ligaments and nerves. In addition, it may provide specific information on bone and cartilage. In this article, we review the use of US in a variety of musculoskeletal applications beyond the hip, including sports injuries, infectious diseases, inflammatory and degenerative conditions, congenital and developmental disorders, acute trauma of bone and joints, and peripheral nerve injuries.

\section{Sports-related injuries}

Apophyseal injuries

In children and adolescents, apophyseal injuries are commonly related to sporting and recreational activities. Especially in the age range between 12 and 16 years, the ossification centres of most apophyses are still immature and the cartilage, under hormonal influence, loses elasticity and is unable to give firm stability to the osteotendinous junction. Hence, the rough shear or torsion forces exercised upon them by tendons and ligaments can overcome the threshold of cartilage and bone resistance causing fragmen- 
tation or detachment with variable functional impairment. The apophyseal damage derives from a traction mechanism that produces traumatic injury to the cartilage and subchondral bone [3]. Then, there is a sequence of overlapping damage-repair events. Under these circumstances, the diagnosis influences the decision with respect to the treatment strategy and the resumption of sporting activity. At the site of insertion into the apophysis, two main categories of tendon lesions may occur: chronic and acute. The first is caused by microtrauma from repeated traction, often in relation to functional overload (overuse). These injuries lead to fragmentation at the tendon-to-bone interface. The degree of avulsion of apophyseal structures is minimal and does not significantly compromise the biomechanics. When detachment involves fragments of cartilage, these can later become ossified, forming small foci of heterotopic ossification; the distance intervening between these ossicles and the apophysis is limited and usually filled with fibrocartilage or with a fibrous bridge. If the displacement is short and the ossicles in close contact with the apophyseal cartilage, they are resorbed into the apophysis during bone maturation. In these cases, a residual prominence or deformity may be found. When the fragment is instead dislocated away from the growing apophysis, it can be transformed into a permanent ossicle.

Acute apophyseal injury is caused by a single unique indirect major trauma. In these injuries, the tendon applies an excessive traction force that is excessive relative to the resistance posed by an apophysis that is not fully mature and biomechanically consolidated. The pelvis is one of the most common sites for acute apophyseal injuries [4, 5]. The most frequently injured apophyses in the pelvis are: the secondary ossification centre of the iliac crest (abdominal wall muscles); the anterior superior iliac spine (sartorius and tensor fasciae latae); the anterior inferior iliac spine (rectus femoris); the pubis (adductors); the ischial tuberosity (hamstrings); and the lesser trochanter (iliopsoas). The elbow is also commonly involved with apophyseal injuries at the medial (flexor origin) and the lateral (extensor origin) epicondyles. The injury leads to partial or complete detachment of the apophysis onto which the tendon is attached. Acute apophyseal injuries can be subdivided into major and minor based on the degree of dislocation of the apophyseal fragment [6]. Major apophyseal lesions cause dislocation of the avulsed fragment at an extent that precludes resorption. Growth may become compromised or arrested, and, in cases of complete avulsion, there will be a significant biomechanical deficit. When traction is insufficient for causing complete avulsion of the apophysis, the fragmentation pattern can differ, involving variable amounts of cartilage and bone. The imaging pattern of acute apophyseal lesions may vary based on the amount of detached bone and cartilage (Figs. 1 and 2). Compared with MRI, US has the advantage of being a clinical examination guided by the patient's symptoms, and being able to identify even minimal cartilage and bone abnormalities. In minor apophyseal injuries, US used as a complement to radiographic examinations can be adequate for diagnosis and characterisation, thus limiting MRI to evaluation of major lesions. Unlike acute injuries, chronic apophyseal lesions are common at the knee (e.g. Osgood-Schlatter and Sinding-Larsen-Johansson disease) and ankle (e.g. Sever disease, accessory navicular syndrome) [7, 8]. Important US signs include fragmentation of the secondary ossification centre, thickening and echotextural abnormalities of the tendon insertion, and local soft-tissue oedema. In these syndromes, US is used during exacerbation of symptoms to study the status of the tendon insertion $[8,9]$.

\section{Ligament injuries}

In children, ligaments are more lax than in adults, and joints hypermobile. The ligament laxity may be further increased in girls after puberty as a result of hormonal influence. Ligament laxity is in itself protective for the ligament, but instability is unfavourable for the joint. In general, injuries in children involve the ligament attachments into the immature bone rather than the ligament itself. Although all ligaments can theoretically be injured, most lesions involve the lateral ankle ligaments following inversion sprains. The anterior talofibular ligament is the single most commonly damaged. Typically, US demonstrates a small osteochondral avulsion from one of the ligament insertions (Fig. 3) [10]. Fragments are often entirely chondral and cannot be seen on plain films. The fate of avulsed fragments is twofold: if their position is adjacent to the avulsion site, new-formed bone will fill the gap resorbing the avulsion; if the fragment is too displaced, progressive ossification and growth of the fragment within the ligament can form an ossicle. In most instances, the diagnostic work-up is based on US alone and does not require other imaging.

Sprain of the medial collateral ligament of the elbow and the knee is common in sporting activities in children and adolescents. The "little league elbow syndrome" is a valgus overload injury to the medial elbow that occurs as a result of repetitive throwing motion [11]. This condition typically affects adolescent baseball pitchers. Valgus stress applies tension to the medial aspect of the elbow joint, including the medial epicondyle, the medial epicondylar apophysis, and the anterior band of the medial collateral ligament, producing ligament injury or medial epicondyle avulsion fracture. Secondary compression of the radiocapitellar joint may concur and potentially cause complications (e.g. osteochondritis dissecans and Panner disease). US can provide accurate evaluation of the medial collateral ligament and the medial epicondylar apophysis [11]. Dynamic imaging during valgus stress may evaluate the stability of the 

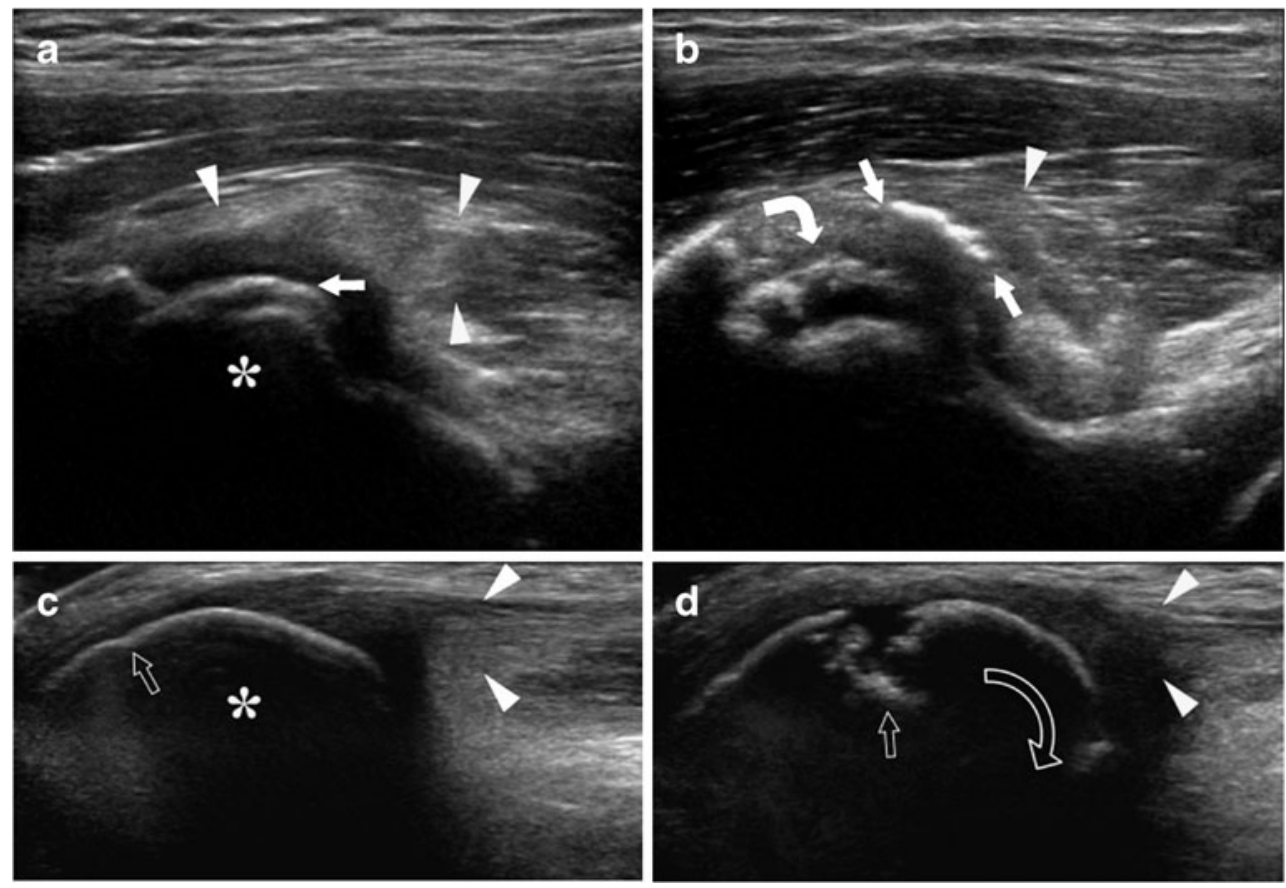

Fig. 1 Apophyseal injuries. a, b Avulsion injury of the anterior inferior iliac spine. a Sagittal US image of the normal anterior inferior iliac spine (asterisk) on the normal side shows the tendon (arrowheads) of the rectus femoris muscle arising from an apophyseal surface that is still cartilaginous. The apophysis has a flattened ossification centre (arrow). b On the injured side, avulsion and rotation of the secondary ossification centre (arrows) is seen in continuity with the tendon of the rectus femoris (arrowhead). Between the avulsed nucleus and the base of the apophysis,

trochlea-ulna joint and give information about partial thickness tears, in which the ligament is continuous but lax [12]. Comparison with the non-injured side should be made to compare the amount of joint widening that occurs during valgus stressing [11, 12]. US is also able to identify medial epicondylar fragmentation, osteochondritis dissecans of the capitellum, and loose bodies [13].

\section{Infection}

Acute osteomyelitis

In acute osteomyelitis, US can identify early abnormalities in the soft tissues overlying the bone as early as 2 days after the onset of symptoms, before any radiographically visible abnormality $[14,15]$. As the infection progresses, the main US signs of osteomyelitis include: oedematous swelling of the soft tissues adjacent to the bone (after 1-3 days); a thin layer of subperiosteal fluid causing detachment and elevation of the periosteum (if the periosteum is strongly attached, the fluid may be extraperiosteal); cortical erosions (after 24 weeks); formation of abscesses and possible spread of heterogeneous cartilaginous tissue (curved arrow) due to haemorrhage and local oedema is seen. c, d Avulsion injury of the tuberosity of the navicular. c Sagittal US image of the normal insertion of the tibialis posterior (arrowheads) into the tuberosity of the navicular. Note the level of fusion (arrow) of the navicular with the secondary ossification centre of the tuberosity (asterisk). d On the injured side, avulsion (straight arrow) and rotation (curved arrow) of the tuberosity of the navicular due to traction by the tibialis posterior tendon (arrowheads) is seen

infection from bone to joint when the metaphysis is intracapsular [16-19]. Doppler imaging may reveal a hypervascular pattern within and around the infected periosteum: this finding can be used as a parameter for monitoring the progression or healing of the inflammatory process and assess the response to antimicrobial therapy [20]. Finally, US can guide aspiration of subperiosteal abscesses for culture and drainage $[15,21]$. Although MRI is the most sensitive and specific imaging modality for evaluating bone and extraosseous changes in osteomyelitis, repeated scanning may be restricted by the need of sedation. US can provide useful information for an early diagnosis and can be used to monitor the infectious process during treatment.

\section{Septic arthritis}

In septic arthritis, a delayed diagnosis may result in avascular necrosis and irreversible bone damage, modelling deformities, early osteoarthritis and ankylosis. US is a reliable means to demonstrate early joint changes with effusion, synovial thickening, cartilage damage and periarticular abnormalities (Fig. 4). The echogenicity of an effusion cannot reliably differentiate infectious/non-infectious processes. In fact, 

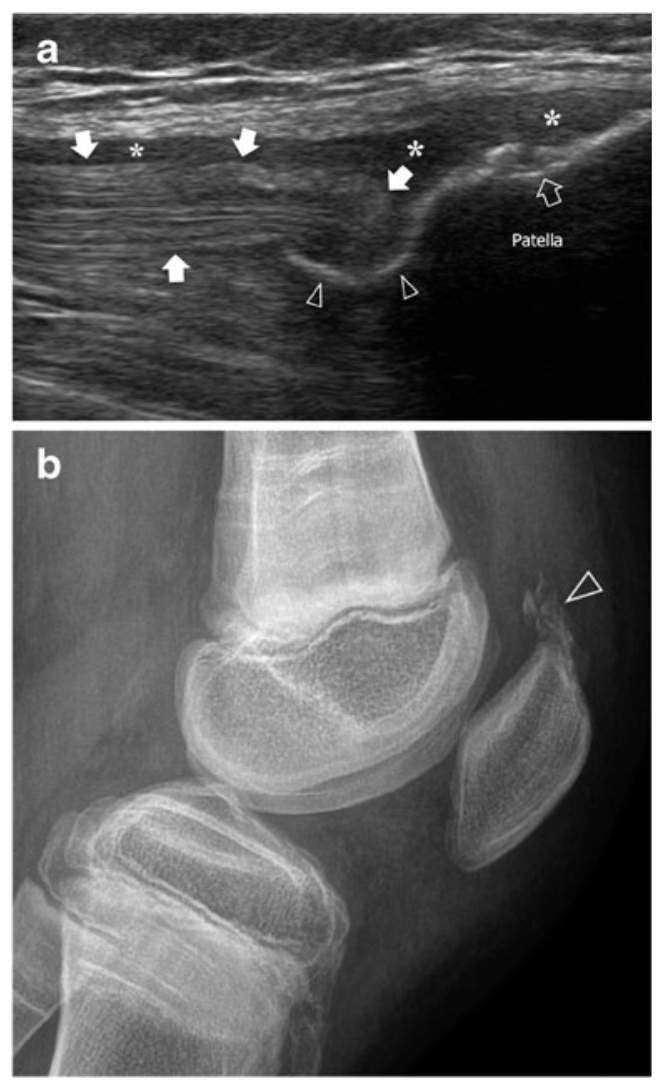

Fig. 2 Patellar avulsion fracture. a Sagittal US at the upper pole of the patella with radiographic correlation (b) reveals a retracted quadriceps tendon (white arrows) attached to a thin bony fragment (arrowheads) displaced from the anterosuperior aspect of the patella. The mild degree of tendon retraction results from the tear not involving the full thickness of the tendon. Note the nidus (open arrow) of the avulsion and the haematoma (asterisks) in the tendinous bed

turbid fluid and debris may also be observed in non-infected joints, and the synovial thickening that accompanies an infection is actually a non-specific finding $[22,23]$. This is why, in cases of suspected septic arthritis, needle aspiration of the effusion remains mandatory, despite causing a number of children with non-infected hips to undergo joint aspiration [24]. Conversely, the absence of a detectable effusion makes the diagnosis of septic arthritis unlikely [25]. US is an excellent modality for guiding the needle into the joint cavity, thus avoiding inadvertent contamination of non-infected spaces, such as bursae and tendon sheaths. Given that most patients with septic arthritis do not show increased flow within and around the joint cavity, Doppler sonography has proved useless for distinguishing infectious/non-infectious synovitis based on hyperaemia [22].

\section{Inflammatory and degenerative arthropathies}

Juvenile idiopathic arthritis

US is an accurate test for intraarticular signs of disease, such as joint effusion, synovial hypertrophy and hyperaemia, and is able to detect subclinical synovitis in children with juvenile idiopathic arthritis [26, 27]. The ability of US to assess multiple joints in one sitting, and to detect subclinical synovitis, seems to be particularly useful in juvenile idiopathic arthritis, since the differentiation of oligoarthritis and polyarthritis is based on the number of inflamed joints. US is therefore ideal for correct classification as well as for suggesting the need for more aggressive treatment and closer monitoring [28]. Based on echotextural characteristics and compressibility, US can differentiate hypertrophied synovium from fluid (Fig. 5). In terms of reliability, preliminary experience revealed moderate to good interobserver agreement in the wrist in patients with juvenile idiopathic arthritis using semiquantitative systems to evaluate synovitis and joint effusion. However, the intraand interobserver variation remains to be widely tested. Variability in equipment still poses a significant problem. Power and colour Doppler sonography can provide a measure of disease activity by assessing the microvasculature of the inflamed synovium, with sensitivity higher than the erythrocyte sedimentation rate or C-reactive protein [29]. After therapy, power Doppler normalisation is seen more frequently in early juvenile idiopathic arthritis compared to patients with longstanding disease [30]. Initially, power Doppler US offered greater sensitivity than colour Doppler in assessment of synovial blood flow [31, 32]. However, in newer US machines, the techniques have near-equal sensitivity, and colour Doppler has the advantage of being less prone to artefact, including noise

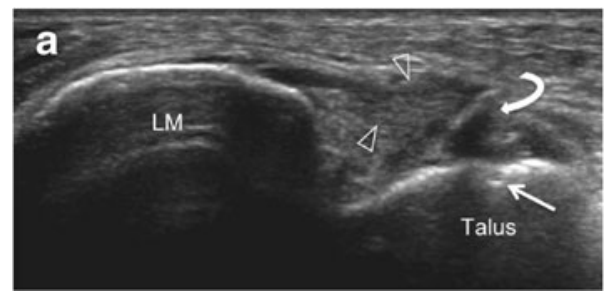

Fig. 3 Anterior talofibular ligament tear. a Long-axis US image over the injured ligament reveals a small flake of bone (curved arrow) in continuity with a wavy hypoechoic thickened ligament (arrowheads).

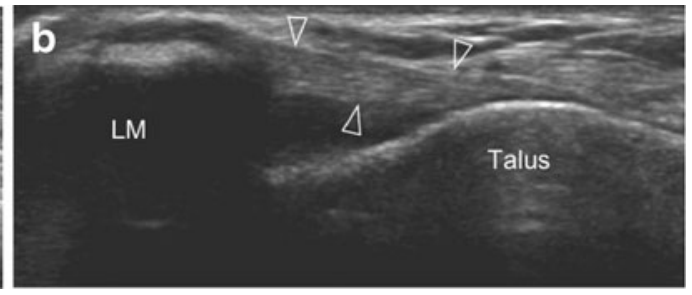

The nidus (straight arrow) in the talus indicates an avulsion injury. $L M$ lateral malleolus. b Normal contralateral ligament (arrowheads) for comparison 

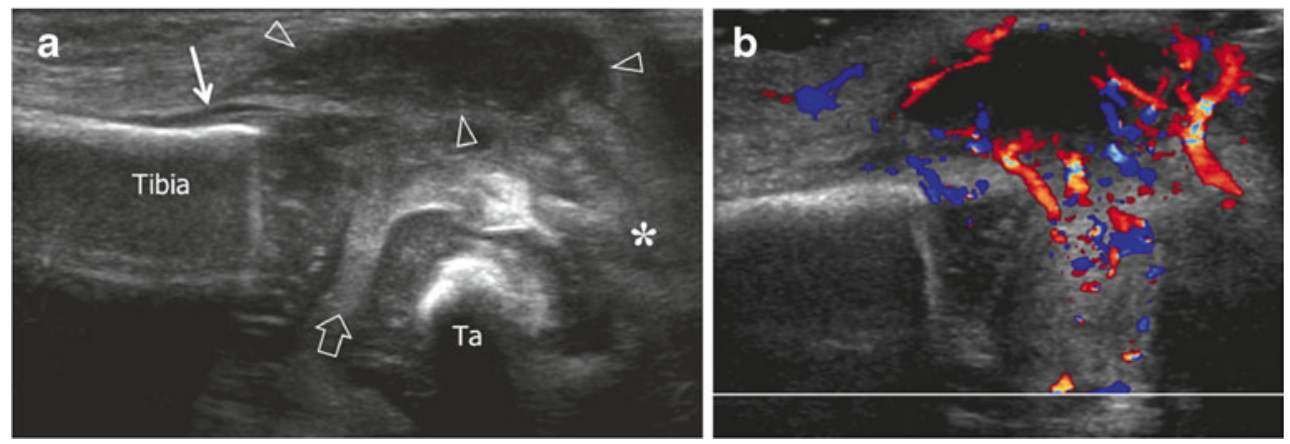

Fig. 4 Septic arthritis in a neonate. a Longitudinal US image at the anterior aspect of the ankle demonstrates purulent echogenic effusion (open arrow) in the tibiotalar joint, associated with periarticular softtissue inflammation (asterisk) and an abscess (arrowheads) in the

and movement [33]. Although contrast-enhanced Doppler sonography has been found to be more accurate than Doppler alone in demonstrating synovial blood flow [34], microbubble-based US contrast agents are still not licensed for use before 18 years of age in Europe and North America. Together with other factors, such as additional cost, time and invasiveness, this limitation makes the use of ultrasonic contrast agents unfeasible.

Cartilage thickening (early disease) or thinning (late disease) with blurring of the normally sharp margins, has been regarded a sign of inflammatory involvement in

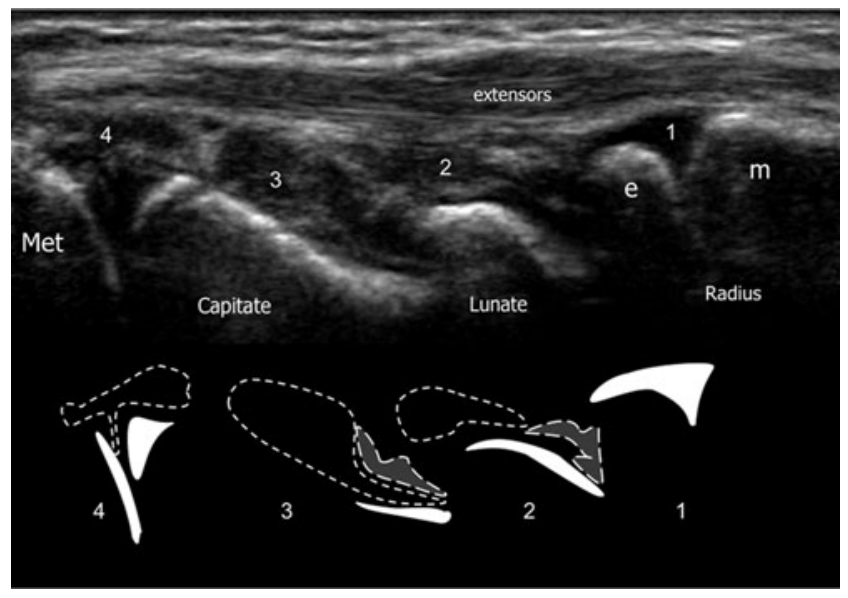

Fig. 5 Juvenile idiopathic arthritis. Midsagittal US image over the dorsal wrist demonstrates the cortical surfaces of the radial metaphysis $(m)$ and epiphysis $(e)$, the lunate, the capitate and the base of the third metacarpal (Met). Superficial to the wrist bones and deep to the extensor digitorum tendons, hypo-/anechoic areas can be found reflecting cartilage (anechoic), synovial pannus (hypoechoic) and joint effusion (anechoic) at the level of the radial epiphysis (1), in the radiocarpal (2), midcarpal (3) and carpometacarpal (4) recesses. These structures can be distinguished based on subtle echotextural differences. In the bottom part of the image, drawings illustrate the extension of cartilage (white), synovial pannus (transparent) and joint effusion (light grey) in each of the areas (1-4) marked in the ultrasonogram anterior superficial tissues. A thin layer of periosteal fluid (thin arrow) is seen superficial to the anterior tibial metaphysis. $T a$ talus. b Corresponding colour Doppler image reveals marked hyperaemia around the joint space and the abscess

juvenile idiopathic arthritis [35]. US measurements of cartilage thickness in target joints of healthy children have been validated against MRI [36, 37]. In addition, age- and sex-specific normal reference intervals for cartilage thickness of the knee, ankle, wrist and finger joints have recently been published [36, 37]. Finally, US is an excellent means to distinguish between articular synovitis and tenosynovitis [38] and to guide the aspiration and injection of steroids into joints, minimising the risk of inadvertent leakage of steroids into the surrounding soft tissues [39]. Further advancement in US technology with introduction of isotropic-voxel volumetric acquisition are opening new perspectives in providing more reliable and accurate quantification of joint volumes, in measuring the fractional amount of synovial pannus, joint effusion and articular cartilage in a given joint recess, and the amount of intraarticular blood flow using Doppler techniques (Fig. 6). In addition, the storage of serial volume datasets of a joint space over time would allow better comparison of findings in longitudinal studies, providing new insights into assessment of the patient outcome and evaluation of the results of therapy.

\section{Haemophilic arthropathy}

US with Doppler has recently been proposed as an alternative to MRI for evaluation of severity and detection of complications in haemophilic arthropathy [40-42]. In under-treated haemophilic patients, acute or subclinical bleeding may occur repeatedly within joints, causing progressive derangement of intraarticular structures and early osteoarthritis. In the chronic stage, US can reliably identify joint space distension by effusion, synovial hypertrophy, bone erosions, subchondral changes and secondary osteoarthritis [41, 43]. Compared with MRI, US is more operator-dependent and is unable to distinguish synovial hypertrophy from intraarticular haemosiderin 
Fig. 6 Juvenile idiopathic arthritis. Three-dimensional rendering from two different viewpoints of the dorsal recesses of the radiocarpal (a) and midcarpal (b) joints in a child with wrist synovitis. Synovial volumes are expressed as red solids. Volumetric acquisition allows automated calculation of each of the synovial volumes (radiocarpal $=0.40 \mathrm{ml}$; midcarpal $=0.18 \mathrm{ml}$ )
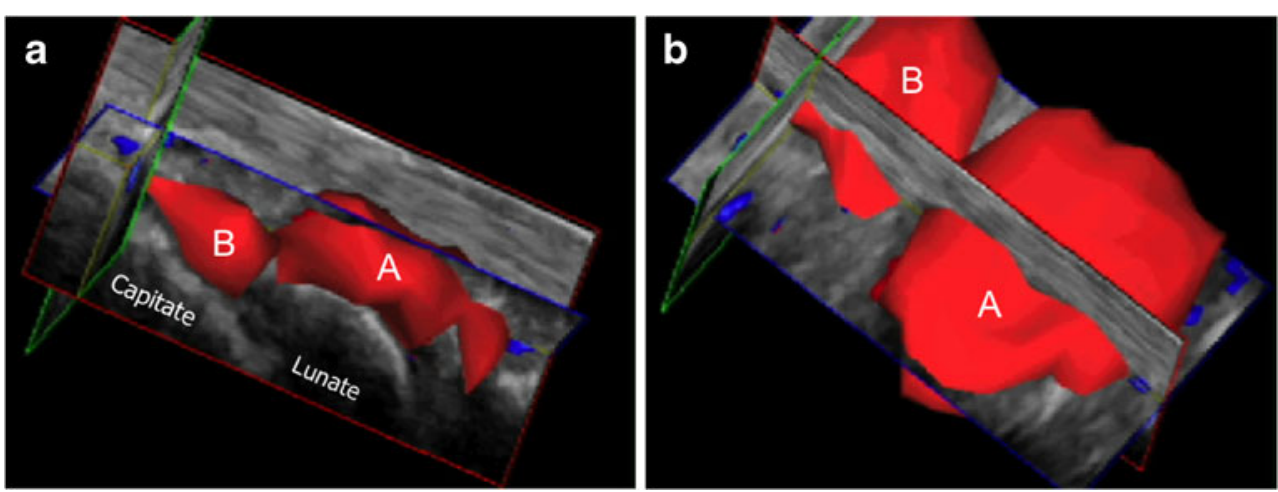

deposition, which is possible with susceptibility-sensitive MRI [44]. US seems particularly helpful in detection of subclinical joint involvement and early signs of joint damage, thus guiding patient management more appropriately. Serial evaluation of the volume and echotexture of haematomas with US makes it possible to follow the progression and regression of haemarthrosis and to determine its age and the status of organisation $[45,46]$. Bleeding may occur into muscles of the lower limb, such as the iliopsoas, quadriceps and gastrocnemius (Fig. 7). In muscles, contractures may result if the haematoma is replaced by scar tissue. In such cases, dynamic US performed during muscle contraction and joint motion may help to assess the extent of the scar and the associated biomechanical deficit. When an intramuscular haematoma does not resolve, it becomes encapsulated and may form a pseudotumour. US may also help the clinician determine the duration of treatment by monitoring the resolution of the haematoma [43].

\section{Congenital and developmental anomalies}

Apart from developmental dysplasia of the hip, there are several congenital and developmental anomalies that involve the musculoskeletal system, where US may be a pivotal or complementary factor in management decision. Based on US assessment, we grouped these disorders into bone, muscle and tendon types.
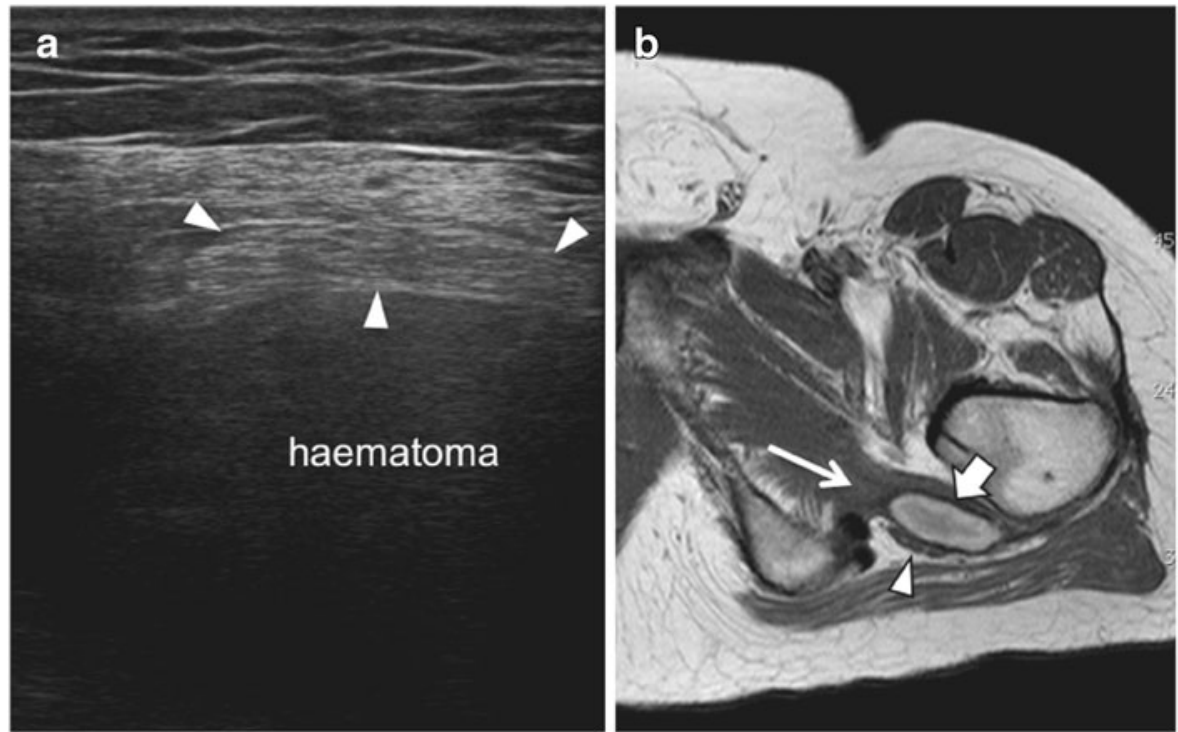

Fig. 7 Haemophilia in a patient with sudden onset of pain and swelling over the left gluteal region, and symptoms of sciatic neuropathy. a Transverse US image reveals a large haematoma that displaces and compresses the sciatic nerve (arrowheads). Corresponding axial T1-weighted (b) and coronal fat-suppressed T2-

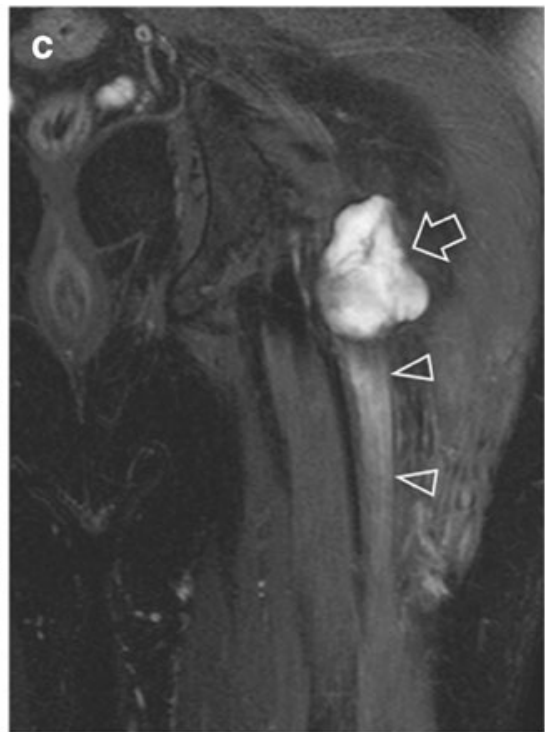

weighted (c) MR images confirm the displacement of the sciatic nerve (arrowhead) by the haematoma (large arrow) located in the quadratus femoris muscle (thin arrow). Note the increased nerve signal intensity on the T2-weighted sequence 


\section{Bone}

In congenital clubfoot, US is a valuable means for estimating the severity of the deformity, and hence helpful for selection of the most appropriate treatment [47-50]. The examination is based on a series of measurements, such as the medial malleolus-navicular distance, the navicular alignment in relation to the talar head, the medial soft-tissue thickness, the talar length and the calcaneocuboid distance [47, 48]. In these patients, the distance between the medial malleolus and the navicular is shorter than normal, and this corresponds to the restricted mobility in the talonavicular joint [48]. In addition, the calcaneo-cuboid distance tends to be increased, leading to medial displacement of the cuboid relative to the calcaneus [48]. On the medial side of the foot, increased soft-tissue thickness as a result of deposition of dense fibrous tissue between the calcaneus and the navicular may be seen on US as a fibrous calcaneonavicular bar, and the tibialis posterior tendon may appear swollen at its insertion. Volumetric US may open new perspectives in quantification of abnormal findings by obtaining information that is unavailable in conventional US, e.g. to measure the talocalcanear angle. Dynamic abduction/adduction stress manoeuvres can be also performed with US [51, 52].
Clubfoot correction with the Ponseti method of manipulation can be monitored quantitatively with US. The progressive realignment of the talonavicular joint can be evaluated with this technique, and inadequate correction can be detected early $[53,54]$. Congenital clubfoot treated with the Ponseti technique may require percutaneous Achilles tenotomy to correct the residual equinus. In these patients, US can effectively assess the Achilles tendon repair [55].

Congenital tarsal coalition is a diagnosis that is often overlooked in children and adolescents. It most often involves the talocalcaneal or talonavicular joints, and presents clinically with foot and ankle pain, and restricted motion. Whereas calcaneonavicular coalitions are readily identified with conventional radiography, talocalcaneal fusion is more difficult to identify on standard radiographic views because of the complex orientation of the subtalar joint. The fusion more commonly involves the middle and posterior facets at the level of the sustentaculum tali and appears as a marked narrowing or closure of the medial joint space that may be displayed with coronal US sections along the medial hindfoot (Fig. 8). In symptomatic patients with suspected congenital tarsal coalition, we believe that US may help exclude such a condition at the talocalcaneal level, avoiding additional
Fig. 8 Congenital talocalcaneal coalition. a Coronal US image at the medial aspect of the normal right hindfoot with radiographic correlation (Harry view, b) demonstrates the normal medial subtalar joint space as a welldefined hypoechoic cortical interval (arrowheads) between the talus $(\mathrm{Ta})$ and calcaneus $(\mathrm{Ca})$. Note the prominence of the sustentaculum tali (asterisk), the medial malleolus $(M M)$ and the position of the tibialis posterior (1), flexor digitorum longus (2) and flexor hallucis longus (3) tendons. c

Corresponding coronal US image over the medial aspect of the affected left hindfoot with radiographic correlation (d) shows closure of the medial subtalar joint space (arrow). The bony walls of the subtalar joint are prominent and irregular
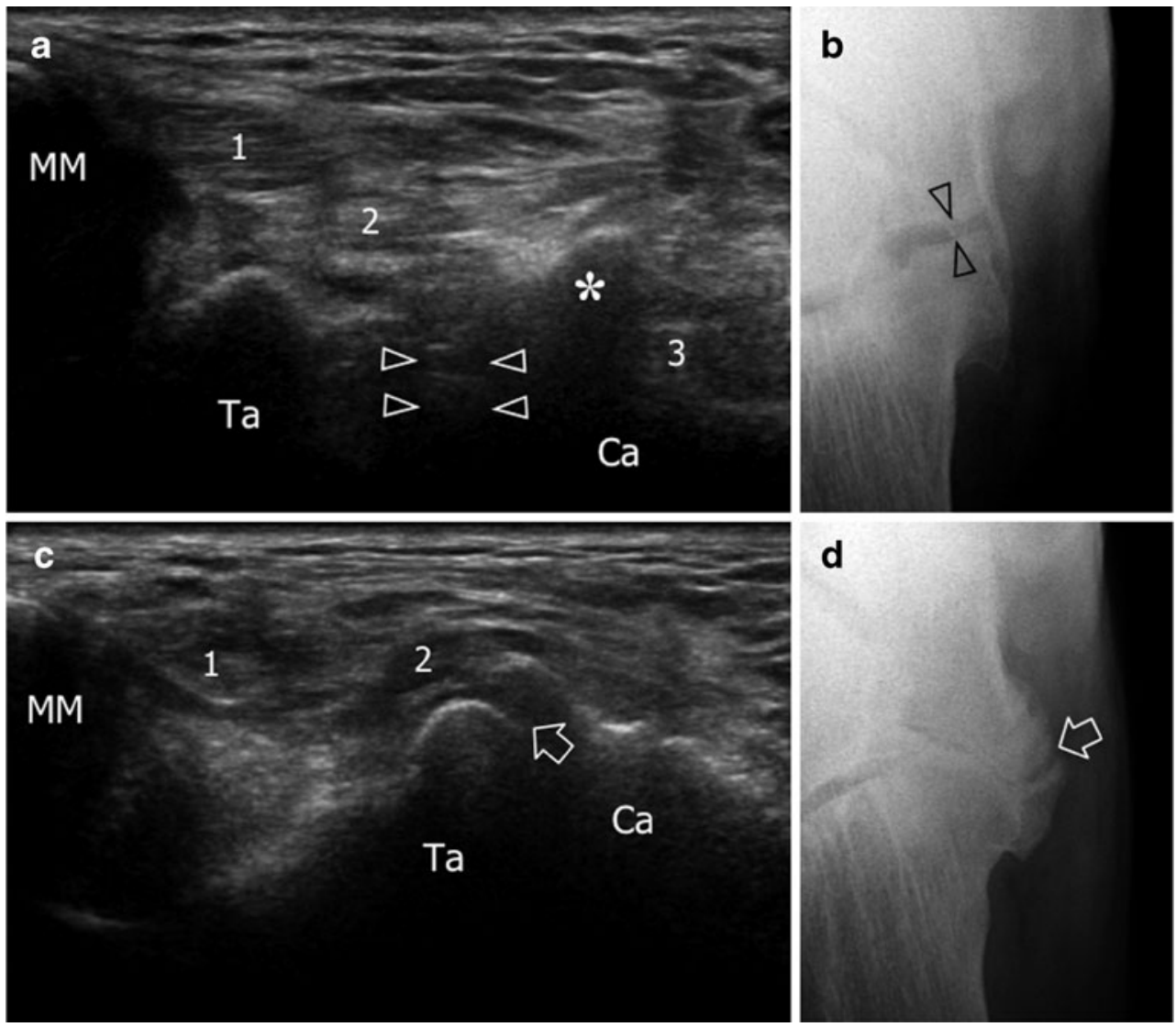
radiographs, CT and/or MRI, which should be reserved for confirmation and preoperative mapping in patients with a positive US examination.

\section{Muscle}

Poland syndrome is a rare congenital aberration related to absence or underdevelopment of the pectoralis muscle complex. The disease is multifaceted and variably associated with anomalies in the chest wall and ipsilateral hand [56]. For patients with Poland syndrome, a variable portion of the pectoralis is missing. The severity of the disorder differs from person to person, and it is often under-diagnosed or under-reported. US can complement clinical examination in assessing abnormalities of the pectoralis complex. It can confirm the diagnosis and distinguish partial forms from complete agenesis of the pectoralis major. In addition, US can easily assess the concurrent agenesis of the pectoralis minor and latissimus dorsi, the latter commonly used for (re-)construction of the pectoralis. Finally, US can complement radiography and $\mathrm{CT}$ in the assessment of anomalies affecting the osseous and chondral part of the rib cage.

In addition to clinical and neurophysiological assessment, neuromuscular imaging is becoming an increasingly important diagnostic tool in inherited muscle disease to detect muscle involvement and describe the severity and pattern of involvement [57]. In this heterogeneous class of disorders, US has proved effective for measuring the muscle bulk. Semiquantitative scales based on muscle echogenicity have been used to estimate dystrophic changes in terms of fatty degeneration and connective tissue replacement [58-60]. In this class of disorders, US allows dynamic scanning during muscle contraction and may reveal spontaneous muscle activity (e.g. fasciculation). Inherent limitations of US are evaluation of deep muscles, complex patterns of involvement, and the relatively high inter- and intraobserver variability [61]. Although some attempts have recently been made to obtain a more reliable quantification of muscle abnormalities in Duchenne and Becker dystrophies by post processing (e.g. backscatter analysis, and entropy-based signal receipt), most of the current imaging experience in dystrophies is based on MRI $[57,62,63]$.

Tendon

A wide array of anatomical variants exists in the musculoskeletal system, including absence of muscles and tendons, supernumerary bellies, anomalous tendon origin and insertion, and muscle deviation. These anomalies result from altered development, or they may represent suppression of the genetic code of atavistic structures [64]. US is an excellent modality for mapping anatomical variants and for assessing altered biomechanics.

Among tendon anomalies, the congenital inability to flex the interphalangeal joint of the thumb is a rare condition consisting of the absence/hypoplasia of the flexor pollicis longus tendon, possibly associated with thumb hypoplasia and absence of the thenar muscles $[65,66]$. US has proved an excellent modality to confirm absence of the tendon and to exclude other differential diagnoses, including an anomalous tendon insertion, congenital tenosynovitis of the flexor pollicis longus, partial anterior interosseous neuropathy and traumatic tendon rupture [67].

The snapping hip has been defined as a syndrome of hip pain accompanied by an audible or palpable clicking or snapping during joint motion [68]. The causes may be extra- or intraarticular. Dynamic US is useful for evaluation of extraarticular causes. In most instances, the syndrome is derived from snapping of the iliopsoas tendon and muscle over the iliopectineal eminence (internal) or catching of the iliotibial band on the greater trochanter (external) [68-71]. Some discrepancy between tendon length and bone maturation is believed to predispose an individual to development of this syndrome. In iliopsoas instability, US images oriented along the short-axis of the tendon are obtained over the iliopectineal eminence while the patient is asked to place the hip in the frog leg position or to generate the hip movement that produces the snapping sensation [68]. An abrupt medialto-lateral or lateral-to-medial motion of the tendon can be observed in real-time. In iliotibial band instability, dynamic US scanning can easily disclose the sudden displacement of

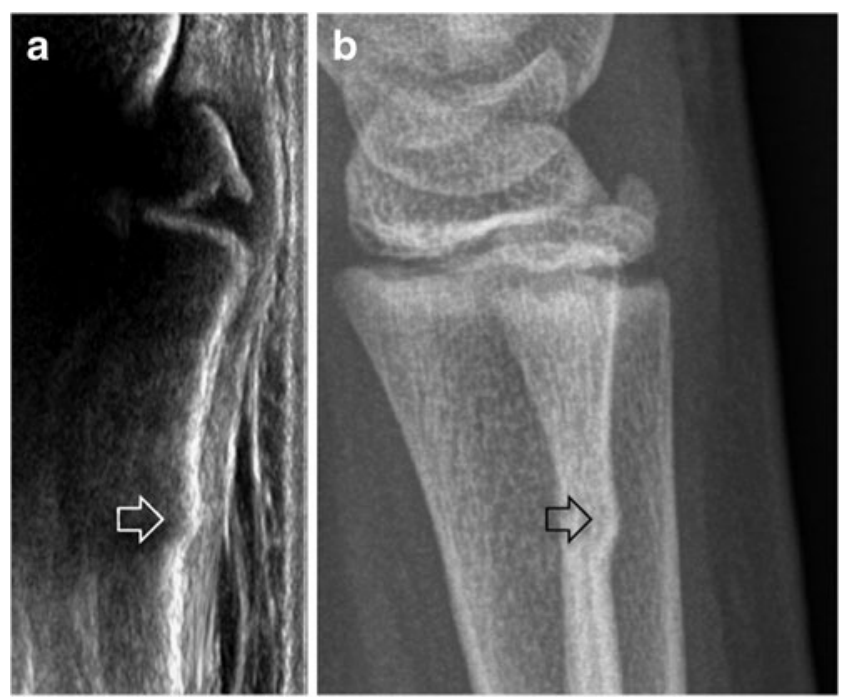

Fig. 9 Distal radial torus fracture in a child first referred for US after a fall on the outstretched arm. a Longitudinal US image in the volar aspect of the distal radius depicts a buckled cortex (arrow) reflecting a torus fracture. b Lateral radiography confirmed the bone injury 
Fig. 10 Fracture separation of the right distal humeral epiphysis in a neonate. a Plain film reveals an abnormal relationship of the radioulnar complex relative to the humeral metaphysis, an appearance consistent with elbow dislocation but also with fracture separation of the distal humeral epiphysis. b Long-axis US image over the radiocapitellar joint demonstrates posterior displacement (dashed line) of the cartilaginous humeral epiphysis (2) from the ossified humeral metaphysis (1). The relationship between the humeral epiphysis and radial head (3) is normal. c Long-axis US image over the left radiocapitellar joint shows the normal alignment for comparison
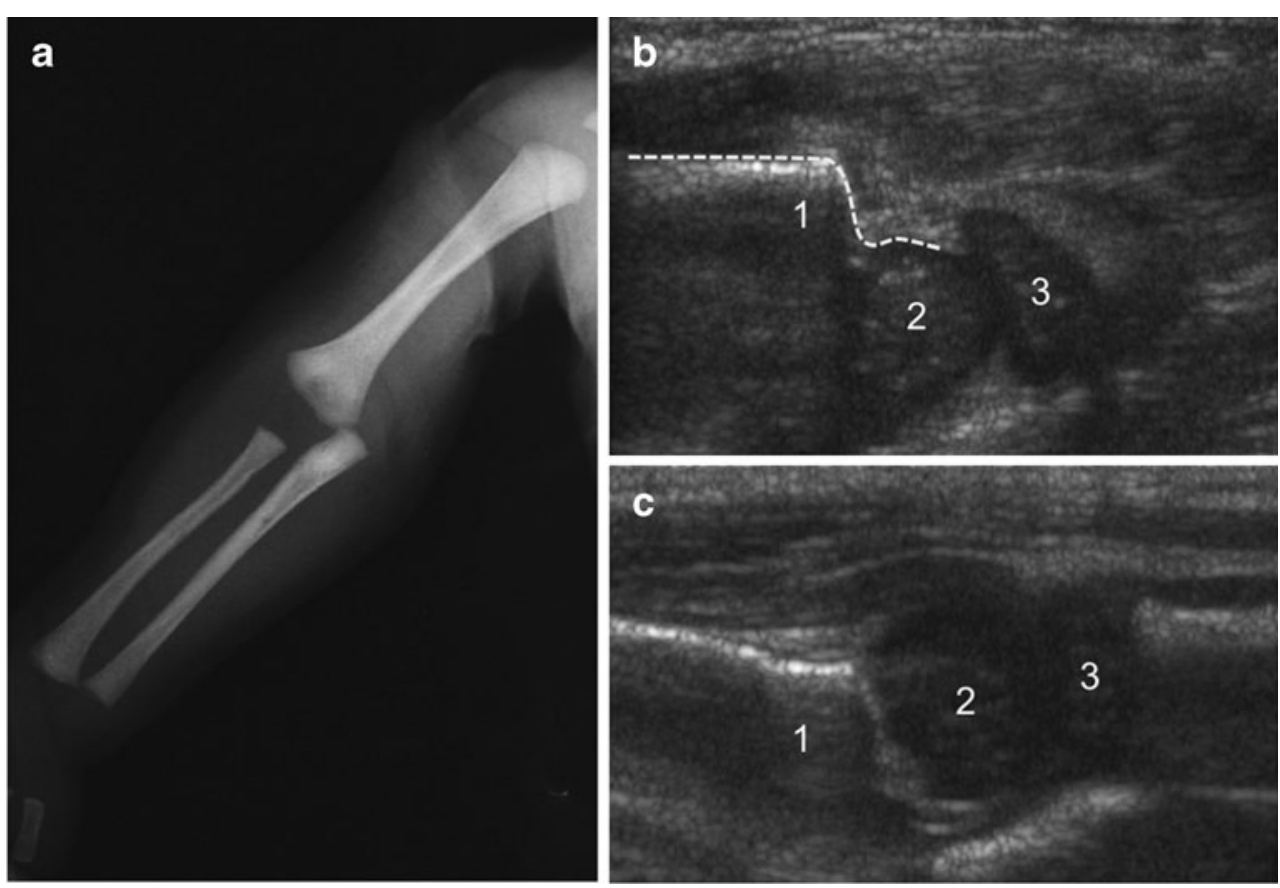

the fascia lata, which mostly occurs when the adducted and extended hip is flexed or when the adducted and internally rotated hip is flexed and externally rotated with the knee bent [71]. US can detect echotextural abnormalities of the fascia lata that may appear thickened and hypoechoic [71]. It is essential to correlate the timing of tendon snapping visible at US with the snapping sensation and, even more importantly, with the referred pain.

\section{Acute bone and joint trauma}

\section{Occult fractures}

It is beyond doubt that the diagnostic work-up for fractures relies on clinical findings and standard radiographs rather than on US. Nevertheless, the anatomical complexity in some regions, and the immaturity of bone in children, may render some fractures undetectable radiographically, especially at the level of the epiphyses. In this setting, US may detect occult fractures incidentally during a conventional US examination of soft tissues. For this reason, bone should routinely be assessed, because even subtle irregularities of the cortex and periosteum may suggest fracture. US seems helpful to detect impacted (torus) fractures, especially when involving the developing metaphyses of the phalanges [72, 73]. These fractures are very subtle and may easily be missed radiographically. On US, these fractures appear as an abnormal metaphyseal bone buckle without interruption of the cortical continuity (Fig. 9). In the acute setting, US has also proved a useful tool for confirming or excluding a variety of fractures around the elbow, including supra-

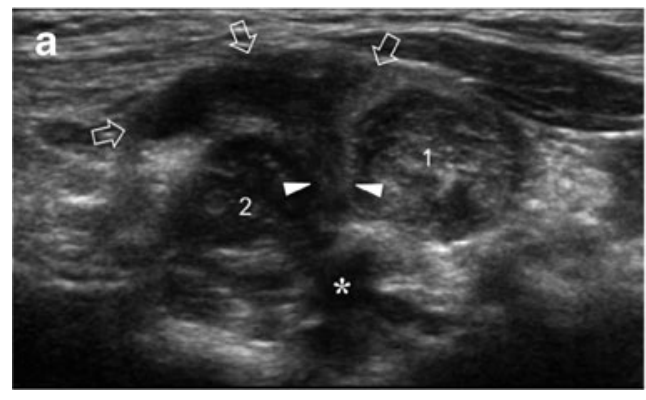

Fig. 11 Interscalenic neuromas of the right brachial plexus in a neonate with obstetric palsy. a Between the anterior (1) and middle (2) scalene muscles, a transverse US image shows a heterogeneous Tshaped mass (arrows) with a blunted proximal end (arrowheads) reflecting retracted nerve roots. b Axial T1-weighted spin-echo MR

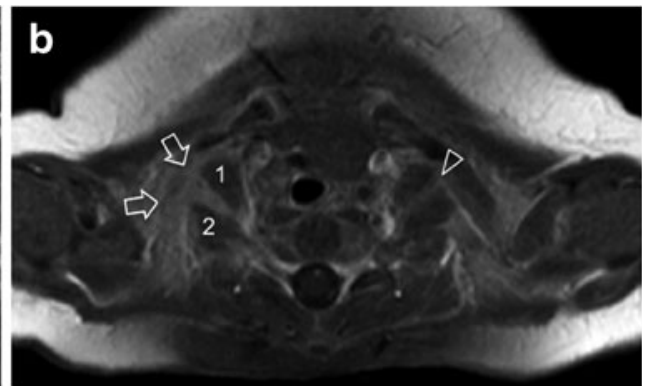

image after intravenous administration of a gadolinium chelate confirms the presence of a hyperintense neuroma of the upper trunk (arrows) intervening between the scalene muscles. Note the normal brachial plexus nerves (arrowhead) at the left interscalenic space for comparison 
condylar, radial neck and distal humeral epiphyseal fractures in neonates and infants, in whom ossification is incomplete [74-76]. In these cases, a fracture may be suspected based on either a cortical step, an echogenic (blood and debris) effusion in the joint space, or separation along a physeal fracture of the chondral epiphysis (hypoechoic) from the bony metaphysis (hyperechoic) (Fig. 10) [77]. Similarly, US may be helpful for distinguishing dislocation of the entire bone (including its unossified epiphysis and diaphysis) from a joint, and a displaced Salter-Harris fracture of the epiphysis (slipped epiphysis) [78, 79].

Pulled elbow

In the subluxated radial head, a condition commonly referred to as "pulled elbow", the annular ligament slips over the radial head when the forearm is pronated. Reduction is commonly achieved without anaesthesia and does not actually require imaging. However, US can identify this condition by measuring the increased radiocapitellar distance during pronation and supination and can distinguish between radial head subluxation with annular ligament interposition (type I) and radial head subluxation with annular ligament tear (type II) [80, 81]. Dynamic US is also able to show restricted radial head rotation during pronation and supination, and the recovery of full range of movement following reduction [81]. In addition, US may be helpful in assessing radiocapitellar joint instability in children whose injury has recurred more than two or three times. In these cases, a dynamic study may depict increased antero-posterior translation of the radial head during pronation and supination [81].

\section{Nerve injuries}

\section{Birth trauma}

In the brachial plexus of newborns and infants, the nerve roots, trunks, divisions and cords can be seen with US at the level of the paravertebral area, interscalenic triangle, supraclavicular region, retropectoralis minor space and axillary area. Traction and compression forces during birth may cause nerve injuries at any of these levels and cause palsy. Adequate surgical planning in these patients requires differentiating preganglionic lesions of the nerve rootlets from postganglionic injures. US has some advantages over MRI in identifying and correctly locating postganglionic injuries occurring outside the neural foramina, limiting the use of MRI to assessment of the cervical spine. Even when US examination is difficult to perform (unco-operative patient), meticulous scanning can effectively rule out abnormalities along the nerves. In obstetric palsy, two characteristic findings can be identified by US: traumatic neuromas and pseudomeningoceles. Neuromas typically involve the nerve roots and trunks in the interscalenic region or slightly distal to it, and appear as bulbous or fusiform hypoechoic masses (Fig. 11). These are the hallmarks of extraspinal brachial plexus injuries, and may be either in continuity with the nerve of origin, or result from retraction of an avulsed nerve root. Pseudomeningoceles appear as hypo-/anechoic collections in proximity to intervertebral foramina. US effectively detects neuromas, but has low sensitivity for pseudomeningoceles. US is promising for demonstrating lesions outside the spinal canal and for establishing the site and level of nerve involvement.

\section{Peripheral nerve injuries}

In addition to brachial plexus trauma, US is excellent for examining injured nerves in the limbs and extremities, and preoperatively it provides information about nerve continuity and the level of nerve transection [82]. In penetrating injuries with partial or complete nerve transection, detection of terminal neuromas may be useful for mapping the location of the nerve stumps and measuring the gap. In stretching injuries, a fusiform hypoechoic swelling of the affected nerve with loss of the fascicular echotexture can be seen, reflecting a spindle neuroma. Scar encasement of the nerve can be encountered when a nerve runs close to a fracture (e.g. median neuropathy secondary to a supracondylar humerus fracture, radial neuropathy in mid-shaft humerus fracture). In these cases, US may help select the appropriate treatment and may provide an accurate preoperative guide for surgical release.

\section{References}

1. Keller MS (2005) Musculoskeletal sonography in the neonate and infant. Pediatr Radiol 35:1167-1173

2. Wilson D (2005) Paediatric musculoskeletal disease: with an emphasis on ultrasound. Springer, Heidelberg

3. Hogan KA, Gross RH (2003) Overuse injuries in pediatric athletes. Orthop Clin N Am 34:405-415

4. Sanders TG, Zlatkin MB (2008) Avulsion injuries of the pelvis. Semin Musculoskelet Radiol 12:42-53

5. Rossi F, Dragoni S (2001) Acute avulsion fractures of the pelvis in adolescent competitive athletes: prevalence, location and sports distribution of 203 cases collected. Skeletal Radiol 30:127-131

6. Stevens MA, El-Koury GY, Kathol MH et al (1999) Imaging features of avulsion injuries. Radiographics 19:655-672

7. De Flaviis L, Nessi R, Scaglione P et al (1989) Ultrasonic diagnosis of Osgood-Schlatter and Sinding-Larsen-Johansson diseases of the knee. Skeletal Radiol 18:193-197

8. Lanning P, Heikkinen E (1991) Ultrasonic features of the OsgoodSchlatter lesion. J Pediatr Orthop 11:538-540

9. Pisacano RM, Miller TT (2003) Comparing sonography with MR imaging of apophyseal injuries of the pelvis in four boys. AJR Am J Roentgenol 181:223-230 
10. Farley FA, Kuhns L, Jacobson JA et al (2001) Ultrasound examination of ankle injuries in children. J Pediatr Orthop 21:604-607

11. De Smet AA, Winter TC, Best TM et al (2002) Dynamic sonography with valgus stress to assess elbow ulnar collateral ligament injury in baseball pitchers. Skeletal Radiol 31:671676

12. Nazarian LN, McShane JM, Ciccotti MC et al (2003) Dynamic US of the anterior band of the ulnar collateral ligament of the elbow in asymptomatic major league baseball pitchers. Radiology 227:149-154

13. Harada M, Takahara M, Sasaki J et al (2006) Using sonography for the early detection of elbow injuries among young baseball players. AJR Am J Roentgenol 187:1436-1441

14. Harcke HT, Grissom LE, Finkelstein MS (1988) Evaluation of the musculoskeletal system with sonography. AJR Am J Roentgenol $150: 1253-1261$

15. Offiah AC (2006) Acute osteomyelitis, septic arthritis and disciitis: differences between neonates and older children. Eur J Radiol 60:221-233

16. Kaiser S, Rosenborg M (1994) Early detection of subperiosteal abscesses by ultrasonography: a means for further successful treatment in pediatric osteomyelitis. Pediatr Radiol 24:336-339

17. Riebel TW, Nasir R, Nazarenko O (1996) The value of sonography in the detection of osteomyelitis. Pediatr Radiol 26:291-297

18. Sammak B, Abd El Bagi M, Al Shahed M et al (1999) Osteomyelitis: a review of currently used imaging techniques. Eur Radiol 9:894-900

19. Bohndorf K (2004) Infection of the appendicular skeleton. Eur Radiol 14:53-63

20. Chao HC, Lin SJ, Huang YC et al (1999) Color Doppler ultrasonographic evaluation of osteomyelitis in children. J Ultrasound Med 18:729-734

21. Larcos G, Antico VF, Cormick W et al (1994) How useful is ultrasonography in suspected acute osteomyelitis? J Ultrasound Med 13:707-709

22. Strouse PJ, DiPietro MA, Adler RS (1998) Pediatric hip effusions: evaluation with power Doppler sonography. Radiology 206:731-735

23. Eich GF, Superti-Furga A, Umbricht FS et al (1999) The painful hip: evaluation of criteria for clinical decision-making. Eur $\mathrm{J}$ Pediatr 158:923-928

24. Bureau NJ, Ali SS, Chhem RK et al (1998) Ultrasound of musculoskeletal infections. Semin Musculoskelet Radiol 2:299-306

25. Dorr U, Zieger M, Haucke H (1988) Ultrasonography of the painful hip. Prospective studies in 204 patients. Pediatr Radiol 19:36-40

26. Magni-Manzoni S, Rossi F, Pistorio A et al (2003) Prognostic factors for radiographic progression, radiographic damage, and disability in juvenile idiopathic arthritis. Arthritis Rheum 48:3509-3517

27. Haslam KE, McCann LJ, Wyatt S et al (2009) The detection of subclinical synovitis by ultrasound in oligoarticular juvenile idiopathic arthritis: a pilot study. Rheumatology 49:123-127

28. Damasio MB, Malattia C, Martini A et al (2010) Synovial and inflammatory diseases in childhood: role of new imaging modalities in the assessment of patients with juvenile idiopathic arthritis. Pediatr Radiol 40:985-998

29. Sparchez M, Fodor D, Miu N (2010) The role of power Doppler ultrasonography in comparison with biological markers in the evaluation of disease activity in juvenile idiopathic arthritis. Med Ultrason 12:97-103

30. Peluso G, Michelutti A, Bosello et al (2011) Clinical and ultrasonographic remission determines different changes of relapse in early and long standing rheumatoid arthritis. Ann Rheum Dis 70:172-175
31. Newman JS, Adlet RS, Bude RO et al (1994) Detection of softtissue hyperemia: value of power Doppler sonography. AJR Am J Roentgenol 163:385-389

32. Walther M, Harms H, Krenn V et al (2001) Correlation of power Doppler sonography with vascularity of the synovial tissue of the knee joint in patients with osteoarthritis and rheumatoid arthritis. Arthritis Rheum 44:331-338

33. Farrant JM, O'Connor PJ, Grainger AJ (2007) Advanced imaging in rheumatoid arthritis. Part 1: synovitis. Skeletal Radiol 36:269-279

34. Fiocco U, Ferro F, Cozzi L (2003) Contrast medium in power Doppler ultrasound for assessment of synovial vascularity: comparison with arthroscopy. J Rheumatol 30:2170-2176

35. Sureda D, Quiroga S, Arnal C et al (1994) Juvenile rheumatoid arthritis of the knee: evaluation with US. Radiology 190:403406

36. Spannow AH, Pfeiffer-Jensen M, Andersen NT et al (2010) Ultrasonographic measurements of joint cartilage thickness in healthy children: age- and sex-related standard reference values. J Rheumatol 37:2595-2601

37. Spannow AH, Stenboerg E, Pfeiffer-Jensen M et al (2011) Ultrasound and MRI measurements of joint cartilage in healthy children: a validation study. Ultrashall Med 32:S110-116

38. Rooney ME, McAllister C, Burns JF (2009) Ankle disease in juvenile idiopathic arthritis: ultrasound findings in clinically swollen ankles. J Rheumatol 36:1725-1729

39. Scott C, Meiorin S, Filocamo G et al (2010) A reappraisal of intraarticular corticosteroid therapy in juvenile idiopathic arthritis. Clin Exp Rheumatol 28:774-781

40. Zukotynski K, Jarrin J, Babyn PS et al (2007) Sonography for assessment of haemophilic arthropathy in children: a systematic protocol. Haemophilia 13(3):293-304

41. Doria AS (2010) State-of-the-art imaging techniques for the evaluation of haemophilic arthropathy: present and future. Haemophilia 16:107-114

42. Melchiorre D, Linari S, Innocenti $M$ et al (2011) Ultrasound detects joint damage and bleeding in haemophilic arthropathy: a proposal of a score. Haemophilia 17:112-117

43. Khan U, Bogue C, Ungar WJ et al (2010) Cost-effectiveness analysis of different imaging strategies for diagnosis of haemophilic arthropathy. Haemophilia 16(2):322-332

44. MacIachlan J, Gough-Palmer A, Hargunani R et al (2009) Haemophilia imaging: a review. Skeletal Radiol 38:949-957

45. Hermann G, Gilbert MS, Abdelwahab F (1992) Hemophilia: evaluation of musculoskeletal involvement with CT, sonography and MR imaging. AJR Am J Roentgenol 158:119-123

46. Kumari S, Fulco JD, Karayalcin G et al (1979) Gray-scale ultrasound: evaluation of iliopsoas hematomas in hemophiliacs. AJR Am J Roentgenol 133:103-106

47. Aurell Y, Johansson A, Hansson G et al (2002) Ultrasound anatomy in the normal neonatal and infant foot: an anatomic introduction to ultrasound assessment of foot deformities. Eur Radiol 12:2306-2312

48. Aurell Y, Johansson A, Hansson G et al (2002) Ultrasound anatomy in the neonatal clubfoot. Eur Radiol 12:2509-2517

49. Chami M, Daoud A, Maestro M et al (1996) Ultrasound contribution in the analysis of the newborn and infant normal and clubfoot: a preliminary study. Pediatr Radiol 26:298-302

50. El-Adwar KL, Taha Kotb H (2010) The role of ultrasound in clubfoot treatment: correlation with the Pirani score and assessment of the Ponseti method. Clin Orthop Relat Res 468:2495-2506

51. Shiels WE 2nd, Coley BD, Kean J et al (2007) Focused dynamic sonographic examination of the congenital clubfoot. Pediatr Radiol 37:1118-1124

52. Coley BD, Shiels WE 2nd, Kean J et al (2007) Age-dependent dynamic sonographic measurement of pediatric clubfoot. Pediatr Radiol 37:1125-1129 
53. Desai S, Aroojis A, Metha R (2008) Ultrasound evaluation of clubfoot correction during Ponseti treatment: a preliminary report. J Pediatr Orthop 28:53-59

54. Aurell Y, Adriesse H, Johansson A et al (2005) Ultrasound assessment of early clubfoot treatment: a comparison of the Ponseti method and a modified Copenhagen method. J Pediatr Orthop B 14:347-357

55. Maranho DA, Nogueira-Barbosa MH, Simao MN et al (2009) Ultrasonographic evaluation of Achilles tendon repair after percutaneous sectioning for the correction of congenital clubfoot residual equinus. J Pediatr Orthop 29:804-810

56. Mentzel HJ, Seidel J, Saumer D et al (2002) Radiological aspects of the Poland syndrome and implications for treatment: a case study and review. Eur J Pediatr 161:455-459

57. Wattjes MP, Kley RA, Fisher D (2010) Neuromuscular imaging in inherited muscle diseases. Eur Radiol 20:2447-2460

58. Heckmatt JZ, Leeman S, Dubovitz V (1982) Ultrasound imaging in the diagnosis of muscle disease. J Pediatr 101:656-660

59. Pillen S, Verrips A, van Alfen N et al (2007) Quantitative skeletal muscle ultrasound: diagnostic value in childhood neuromuscular disease. Neuromuscul Disord 17:509-516

60. Pillen S, Arts IM, Zwarts MI (2008) Muscle ultrasound in neuromuscular disorders. Muscle Nerve 37:678-693

61. Pillen S, Keimpema M, Bievestein RAJ et al (2006) Skeletal muscle ultrasonography: visual versus quantitative evaluation. Ultrasound Med Biol 32:1315-1321

62. Hughes MS, Marsh JN, Wallace KD et al (2007) Sensitive ultrasonic detection of dystrophic skeletal muscle in patients with Duchenne muscular dystrophy using an entropy-based signal receiver. Ultrasound Med Biol 33:1236-1243

63. Zaidman CM, Connolly AM, Malkus EC et al (2010) Quantitative ultrasound using backscatter analysis in Duchenne and Becker muscular dystrophy. Neuromuscul Disord 20:805-809

64. Martinoli C, Perez MM, Padua L et al (2010) Muscle variants of the upper and lower limb (with anatomical correlation). Semin Musculoskelet Radiol 14:106-121

65. Alicioglu B (2007) Radiologic findings of the flexor pollicis longus hypoplasia. Skeletal Radiol 36:120-123

66. Demirseren ME, Afandiyev K, Durgun M et al (2007) Congenital absence of flexor pollicis longus tendon without associated anomalies of thumb hypoplasia: a case report and review of the literature. Hand 2:184-187

67. Suresh SS, Abraham R, Kumar Santhosh S (2009) Absent flexor pollicis longus: case report with review of literature. Hand 4:74-77
68. Pelsser V, Cardinal E, Hobden R et al (2001) Extraarticular snapping hip: sonographic findings. AJR Am J Roentgenol 176:67-73

69. Deslandes M, Guillin R, Cardinal E et al (2008) The snapping iliopsoas tendon: new mechanisms using dynamic sonography. AJR Am J Roentgenol 190:576-581

70. Guillin R, Cardinal E, Bureau NJ (2009) Sonographic anatomy and dynamic study of the normal iliopsoas musculotendinous junction. AJR Am J Roentgenol 19:995-1001

71. Choi YS, Lee SM, Byet S et al (2002) Dynamic sonography of external snapping hip syndrome. J Ultrasound Med 21:753758

72. Simanovsky N, Lamdan R, Hiller N et al (2009) Sonographic detection of radiologically occult fractures in pediatric ankle and wrist injuries. J Pediatr Orthop 29:142-145

73. Cho KH, Lee SM, Lee YH et al (2010) Ultrasound diagnosis of either an occult or missed fracture of an extremity in pediatricaged children. Korean J Radiol 11:84-94

74. Davidson RS, Markovitz RI, Dormans J et al (1994) Ultrasonographic evaluation of the elbow in infants and young children after suspected trauma. J Bone Jt Surg 76:1804-1812

75. Brown J, Eustace S (1997) Neonatal transphyseal supracondylar fracture detected by ultrasound. Pediatr Emerg Care 13:410-412

76. Lazar RD, Waters PM, Jaramillo D (1998) The use of ultrasonography in the diagnosis of occult fracture of the radial neck. $\mathrm{J}$ Bone Jt Surg 80:1361-1364

77. Major NM, Crawford ST (2002) Elbow effusions in trauma in adults and children: is there an occult fracture? AJR Am J Roentgenol 178:413-418

78. Eliahou R, Simanovsky N, Hiller N et al (2006) Fractureseparation of the distal femoral epiphysis in a premature neonate. J Ultrasound Med 25:1603-1605

79. Ziv N, Litwin A, Katz K et al (1996) Definitive diagnosis of fracture-separation of the distal humeral epiphysis in neonates by ultrasonography. Pediatr Radiol 26:493-496

80. Kosuwon W, Mahaisavariya B, Saengnipanthkul S et al (1993) Ultrasonography of pulled elbow. J Bone Joint Surg Br 75:421422

81. Diab HS, Hamed MMS, Allam Y (2010) Obscure pathology of pulled elbow: dynamic high-resolution ultrasound-assisted classification. J Child Orthop 4:539-543

82. Tagliafico A, Altafini L, Garello I et al (2010) Traumatic neuropathies: spectrum of imaging findings and postoperative assessment. Semin Musculoskelet Radiol 14:512-522 